\title{
La objeción de conciencia en la atención sanitaria: el marco mexicano
}

\author{
Jorge Álvarez-Díaz ${ }^{1}$
}

\begin{abstract}
Resumen
Con el objetivo de establecer las características generales de la problemática teórica en relación con la objeción de conciencia en la atención sanitaria, así como su posible aplicación en el marco mexicano, el presente trabajo realiza algunas consideraciones filosóficas básicas para comprender quées (y quéno es) la objeción de conciencia, quécaracterísticas debe de tener para considerarla como tal y qué otras figuras pueden intervenir en el complejo mundo de la atención sanitaria. Se finaliza el trabajo exponiendo el marco mexicano y el caso de la interrupción voluntaria del embarazo, a modo de ejemplificación.
\end{abstract}

Palabras clave $\quad$ Conciencia; atención de la salud; bioética; filosofía médica.
Conscientious objection in health care: the Mexican frame

\section{Abstract}

In order to establish the general characteristics of the theoretical problems regarding to the conscientious objection in health care, as well as its possible application within the Mexican framework, this paper makes some basic philosophical considerations to understand what is (and what is not) the conscientious objection, which characteristics must have to consider it like so, and what other figures can take part in the complex world of health care. We end talking about the Mexican frame and the case of voluntary interruption of pregnancy as an exemplification.

Key words: Conscience; health care; bioethics; philosophy medical.

Un problema importante dentro de la Filosofía del Derecho ha sido el justificar la necesidad de la obediencia al Derecho. Han existido muchos intentos a lo largo de la historia filosófica por justificar dicha obediencia, y casi a la par se ha originado la contraparte: esfuerzos doctrinales por justificar la desobediencia al mismo. El discurso democrático moderno y su práctica dejan manifiesto que hay una necesidad del

\footnotetext{
1 Médico Sexólogo Clínico; Magíster en Bioética; Doctorando en el Programa de Ciencias Sociosanitarias y Humanidades Médicas. Universidad Complutense de Madrid, Facultad de Medicina, Departamento de Historia de la Medicina. Madrid, España.
}

respeto a la ley y al Estado. Por otro lado, el discurso que avala la noción de derechos humanos pareciera albergar la idea de que pueden existir ocasiones en que no se quiera hacer caso de un principio jurídico. Hablar acerca de la objeción de conciencia es tocar un tema complejo, que engarza en cierta medida la noción de bioética y derecho, en un área denominada más recientemente como del bioderecho, o una rama biojurídica.

\section{¿Qué se entiende por objeción de conciencia?}

Se han dado muchas definiciones sobre lo que es la objeción de conciencia. Una puede ser "el derecho subjetivo que tiene por objeto lograr la dispensa de un deber jurídico o la exención de responsabilidad cuando el incumplimiento de este deber se ha consumado" $\left({ }^{1}\right)$. Otra puede ser que se trata de la "pretensión pública individual de un imperativo ético personalmente advertido, en colisión con un deber jurídico contenido en la ley o en un contrato por ella tutelado" $\left({ }^{2}\right)$. Una tercera, pudiera ser que corresponde a "la pretensión de incumplir una ley -o desoír un precepto- de na- 
turaleza no religiosa, por motivos religiosos o ideológicos” ${ }^{(3)}$.

¿Y qué se objeta? Se habla de la 'objeción de conciencia' y hay que tener también un sentido de lo que se entiende aquí por conciencia. La 'conciencia' es un término que dice mucho y nada a la vez, si no se explicita en qué sentido se le está manejando. Se le ha estudiado desde ciertos reduccionismos biológicos $\left({ }^{4}\right)$, entendiéndola como el "darse cuenta que se da cuenta de algo" $\left({ }^{5}\right)$, hasta intentando articular conocimientos biológicos y filosóficos $\left(^{6-9}\right)$. Pero, en este tema se está tratando de un aspecto muy específico, de eso que se conoce como 'conciencia': se refiere en el sentido de las propias creencias, de la llamada conciencia moral.

En resumen, la objeción de conciencia consiste en la negativa del individuo para el cumplimiento u obediencia a una norma o imperativo jurídico en alguna materia, por ir en contra de la conciencia. Es decir, el escenario se plantea con un marco jurídico establecido (no hay que objetar si no hay ley) frente a la conciencia de un individuo. Uno de los muchos problemas de enfrentar la pluralidad de la sociedad (expresada en las leyes) a la particularidad (propia de cada ciudadano, de cada individuo). Con ello, en opinión de Ruiz Rodríguez, "la objeción de conciencia le permite al hombre tomar una decisión en el ámbito meramente personal, incluso frente a lo que hayan consensado las mayorías -que es una característica de la democracia-, porque va en contra de sus principios o, dígase, de su moral" $\left({ }^{10}\right)$. La descripción clásica es la objeción a la prestación del servicio militar por razones de conciencia.

De aquí surgen las primeras cuestiones que llevan al análisis y la reflexión de lo complejo de la problemática planteada por la objeción de conciencia. Si el individuo debe de cumplir la ley, ¿debe de cumplirla aún y cuando mande cosas injustas? ¿Con qué criterio puede el individuo hacer el juicio si la ley es injusta o no? Por otro lado, si a lo que se apela es a la conciencia moral del individuo, ¿todo juicio de una conciencia moral individual es respetable por el solo hecho de haber sido elaborado por una persona?, ¿o este juicio es respetable por apelar a valores superiores al individuo que hace el juicio? Estos problemas llevan al análisis de si es posible escindir (y hasta dónde) la moralidad de la legalidad. De hecho, la defensa de la objeción de conciencia da por sentado que existe una parte de la moralidad común que puede, y debe, ser regulada por el derecho; pero existe también una moralidad privada, individual, que puede entrar en conflicto con la anterior.

Por otro lado, también se puede analizar otro tipo de problemas, como pueden ser las discusiones desde el inicio mismo de la cultura en torno al concepto de lo que es la justicia. Las leyes deben ser justas; esta tesis está presente desde los griegos, pero el concepto de justicia tiene desde su origen el problema de ser un concepto formal, y al momento de materializarlo, esto es, de dotarlo de contenido, no todo el mundo entiende lo mismo por lo que es justo. En el mundo de la bioética, esto es muy importante, debido a que la corriente iniciada por Beauchamp y Childress, conocida como principialismo y que ha tenido un eco enorme en el llamado mundo occidental, tiene como uno de los cuatro principios fundamentales de la bioética a la justicia.

También debe ser digno de análisis el problema de los juicios emitidos desde la conciencia moral. Como se ha señalado, "la objeción de conciencia, para ser aceptada como tal, no puede ser producto del capricho o del mero juicio subjetivo del objetor, sino que debe estar basada en una conciencia cierta, recta y bien formada" $\left({ }^{11}\right)$. Este aspecto también es crucial en la bioética principialista, ya que otro principio fundamental es el de la autonomía. Además, recordando lo que proponen Beauchamp y 
Childress, actuar con autonomía no consiste en hacer cumplir el propio deseo, sino que una persona actúa con autonomía cuando tiene independencia respecto de controles externos y capacidad para obrar de acuerdo a una elección propia, juzgando el grado de intencionalidad de los actos, la comprensión que de ellos tiene el agente y la ausencia de coerciones o limitaciones (internas o externas). De esta manera, se puede ver que no es sencillo el elaborar un juicio sobre la autonomía de un agente que quiera recurrir a la objeción de conciencia.

Se puede hacer algunas aclaraciones y acotaciones respecto a la objeción de conciencia en sus aspectos prácticos, ya que no es propósito de este trabajo el agotar o resolver la problemática teórica.

\section{Consideraciones a la objeción de conciencia}

En este momento, ya se tiene el marco conceptual de lo que es una objeción de conciencia y algunos problemas teóricos respecto a tal reflexión. Lo que está un poco más claro es que tiene que existir una ley y alguien que, por algún motivo atribuido a su conciencia moral, no quiera acatar. Esto debe de quedar muy claro, ya no siempre se puede hablar de objeción de conciencia y confundírsela con otras figuras. Además, el análisis de la posibilidad de actuar en objeción de conciencia se debe de hacer de acuerdo al marco legal de cada país, donde solo caben dos posibilidades: la objeción de conciencia se deriva directamente de un derecho fundamental en la Constitución, o bien se debe realizar un análisis particular caso por caso.

Algunas consideraciones que han sido propuestas a modo de guía, para identificar aspectos relevantes en torno a la objeción de conciencia, son las siguientes:

1. Se considera que existe objeción de conciencia cuando no existe un fin político; es decir, el acto se realiza por el verdadero conflicto entre la normativa legal y la conciencia del objetor, no para obstaculizar alguna otra decisión, para influir en la opinión publica o actuar de manera mediática, para generar polémica y ganar adeptos para la postura del objetor, etc.

2. La objeción de conciencia tiene un peso moral cuando se basa en creencias centrales de los valores éticos del profesional sanitario, y tiene un menor peso moral cuando se basa en creencias que son periféricas a tales valores. Las creencias morales centrales en los valores éticos del personal sanitario tienen que ver con la autoimagen y el autoentendimiento y, por ello, con la noción de integridad moral.

3. La objeción de conciencia tiene mayor peso moral cuando tiene como trasfondo las concepciones individuales sobre lo que significa ser un personal sanitario ético; pero tiene menor peso moral cuando el trasfondo consiste en las concepciones individuales del personal de salud respecto a ser una persona generalmente ética, o un miembro ético de un grupo étnico, religioso, cultural u otro.

4. Cada vez que esto sea posible, la objeción de conciencia debe de tener cabida sin comprometer los derechos e intereses de terceros, como lo son los pacientes.

5. Precisamente, como el peso moral de la objeción de conciencia puede variar mucho, los valores e intereses en conflicto pueden tener un peso moral mayor o menor. Es necesario deliberar adecuadamente sobre este tema antes de emitir un juicio por parte del profesional sanitario.

\section{La objeción de conciencia en atención sanitaria}

Existen una serie de condiciones o escenarios en las cuales se puede dar el caso de objetar la conciencia moral. Este es el propósito de exponer las ideas en torno a la 
objeción de conciencia: el apreciar sus posibles aplicaciones en el ámbito sanitario.

Tanto el médico (como cualquier otro profesional sanitario) y el paciente cuentan con autonomía. Vale aclarar que, en opinión de Gracia Guillén, ha sido difícil y reciente la introducción de la noción de autonomía del paciente en la práctica médica $\left({ }^{12}\right)$ : lo tradicional había sido el reconocimiento de la propia autonomía del clínico. Por ello, los dos escenarios que se comentan a continuación, tal vez el primero sea más familiar, en cierto sentido, al hablar de la objeción de conciencia.

\section{1.- Cuando el profesional sanitario recurre a la objeción de conciencia.}

Algunos profesionales sanitarios cuentan con una libertad prescriptiva, como pueden ser los médicos y los farmacéuticos en algunos países. Apelando a ello, el profesional puede recurrir a la objeción de conciencia y negarse a prescribir un medicamento o un tratamiento de otra naturaleza (quirúrgico, por ejemplo).

Escenarios respecto a la posibilidad de recurrir a la objeción de conciencia en este sentido son cuando los profesionales se niegan a vender fármacos, tales como la anticoncepción de emergencia; por otro lado, puede referirse a no realizar un procedimiento quirúrgico, como puede ser un legrado intrauterino o practicar una infibulación a una niña islámica.

\section{2.- Cuando el paciente recurre a la objeción de conciencia.}

También, es bueno recordar que el paciente cuenta con libertad, una libertad terapéutica, ya que en los últimos años se ha reconocido gracias a la introducción el principio de autonomía en bioética, y al reconocimiento de que finalmente el hecho clínico se desarrolla en el cuerpo del paciente, no en el cuerpo del profesional sanita- rio. Esto es, se reconoce también que quien debe de gestionar el cuerpo es la propia persona.

El ejemplo típico podría ser cuando por razones de conciencia moral religiosa no se admite algún procedimiento, médico o quirúrgico, como en el caso de los miembros de religiones pentecostales o testigos de Jehová.

\section{3.- Situaciones donde no se puede considerar la objeción de conciencia.}

Existen algunos casos en donde no se puede hablar de una objeción de conciencia como tal. El mejor ejemplo es cuando se toma decisiones surrogadas en menores de edad (niños y/o adolescentes).

Ya de por sí el tema de las decisiones surrogadas en menores encierra muchos problemas, más cuando se trata de una situación donde se pueda recurrir a la posibilidad de la objeción de conciencia. Un ejemplo típico es el citado cuando los padres, testigos de Jehová, se rehúsan a que su hijo menor de edad sea transfundido "por razones de conciencia'. El gran problema aquí, en relación con la objeción de conciencia, es que el hecho clínico se desarrollará en el cuerpo del menor, quien no tiene la capacidad ni la competencia legal para recurrir a la objeción de conciencia, ya que sería quien podría recurrir a tal figura.

\section{El marco mexicano}

Para el caso mexicano, se puede decir que la Constitución Política de los Estados Unidos Mexicanos, en su artículo $5^{\circ}$, considera como bienes y otorga protección a la vida, la salud y la libertad, estableciendo que «el Estado no puede permitir que se lleve a efecto ningún contrato, pacto o convenio que tenga por objeto el menoscabo, la pérdida o el irrevocable sacrificio de la libertad de la persona por cualquier cau- 
sa». En la tradicionalmente entendida objeción de conciencia, en relación con la libertad religiosa, ésta se consagra en el artículo 24: "Todo hombre es libre para profesar la creencia religiosa que más le agrade y para practicar las ceremonias, devociones o actos del culto respectivo, siempre que no constituyan un delito o falta penados por la ley".

Por otro lado, el artículo $1^{\mathrm{o}}$ de la Ley de Asociaciones Religiosas y Culto Público, en su párrafo segundo establece que "las convicciones religiosas no eximen en ningún caso del cumplimiento de las leyes del país. Nadie podrá alegar motivos religiosos para evadir las responsabilidades y obligaciones prescritas en las leyes". Además, el Código Civil establece que "la voluntad de los particulares no puede eximir de la observancia de la ley, ni alterarla o modificarla. Solo puede renunciarse los derechos privados que no afecten directamente el interés público, cuando la renuncia no perjudique los derechos de tercero" (artículo $6^{\circ}$ ); «la renuncia autorizada en el artículo anterior no produce efecto alguno si no se hace en términos claros y precisos, de tal suerte que no quede duda del derecho al que se renuncia» (artículo $7^{\circ}$ ); "los actos ejecutados contra el tenor de las leyes prohibitivas o de interés público serán nulos, excepto en los casos en que la ley ordene lo contrario" (artículo $8^{\circ}$ ); "contra la observancia de la ley no puede alegarse desuso, costumbre o práctica en contrario" (artículo $\left.10^{\circ}\right)$; y, "las leyes que establecen excepción a las reglas generales no son aplicables a caso alguno que no está expresamente especificado en las mismas leyes" (artículo $11^{\circ}$ ).

La Declaración Universal de los Derechos Humanos, en su artículo 18, dice que «toda persona tiene derecho a la libertad de pensamiento, de conciencia y de religión; este derecho incluye la libertad de cambiar de religión o de creencia, así como la libertad de manifestar su religión o su creencia, individual o colectivamente, tanto en público como en privado, por la enseñanza, la práctica, el culto y la observancia». Es decir, en el fondo el Código Civil parece contravenir este artículo.

Además de estas normativas jurídicas, existen algunas recomendaciones en materia de salud, pero, mas bien, dentro del orden moral (puesto que si no son cumplidas no existe sanción alguna). Una sería la Carta de los Derechos Generales de los Médicos de la Comisión Nacional de Arbitraje Médico (CONAMED), donde no está plasmado un derecho a la objeción de conciencia como tal, pero anota en su primer precepto sobre el derecho a "ejercer la profesión en forma libre y sin presiones de ninguna naturaleza", que se refiere a que "el médico tiene derecho a que se respete su juicio clínico (diagnóstico y terapéutico) y su libertad prescriptiva, así como su probable decisión de declinar la atención de algún paciente, siempre que tales aspectos se sustenten sobre bases éticas, científicas y normativas". En la contraparte, la Carta de los Derechos Generales de los Pacientes, en su cuarto precepto acerca de "decidir libremente sobre su atención", consigna que "el paciente, o en su caso el responsable, tienen derecho a decidir con libertad, de manera personal y sin ninguna forma de presión, aceptar o rechazar cada procedimiento diagnóstico o terapéutico ofrecido, así como el uso de medidas extraordinarias de supervivencia en pacientes terminales".

Otros documentos, ahora de la Comisión Nacional de Bioética, son el Código de Bioética para el Personal de Salud 2002, cuyo capítulo IV consigna "potestades bioéticas del personal de salud", y su artículo 28 dice que "El personal de salud podrá rehusarse a aplicar medidas diagnósticas y terapéuticas que a su juicio pongan en riesgo la vida, la función de los pacientes o su descendencia, bien sea a petición de los propios pacientes, de sus superiores jerárquicos o autoridades institucionales, cuan- 
do se oponga a la práctica médica comúnmente aceptada, a los principios bioéticos, a sus capacidades profesionales o a razones de objeción de conciencia" ( $\left.{ }^{13}\right)$; pero, por otro lado, tiene un Código de Conducta para el Personal de Salud 2002, en cuya primera parte de "estándares de trato profesional" consigna en su artículo 27 que el personal sanitario "se apegará invariablemente a las normas oficiales, así como a los programas, protocolos y procedimientos establecidos en su institución, para el diagnóstico, tratamiento o rehabilitación de los pacientes" $\left({ }^{14}\right)$.

Así pues, en México no se cuenta con una legislación específica respecto a la objeción de conciencia en materia de atención sanitaria, por lo que el tema de la objeción de conciencia debe ser analizado caso por caso.

\section{El ejemplo de la interrupción voluntaria del embarazo (IVE)}

En materia específica de la objeción de conciencia en la IVE, como ejemplo para ilustrar sobre un caso discutido y discutible, se ha interpretado que, desde la Declaración Universal de los Derechos $\mathrm{Hu}$ manos, debe de respetarse tanto la autonomía de una mujer que solicita un servicio de salud del tipo de la IVE, como la autonomía del personal de salud $\left({ }^{15}\right)$. Por ello es que, a pesar de que las creencias personales del personal de salud se opongan a las de la mujer que solicita una IVE, ésta debe de ser tratada con dignidad, respeto y tiene el derecho a un cuidado que no dependa del juicio sobre ella $\left({ }^{16}\right)$. Incluso, se ha mencionado que, uno de los límites para la objeción de conciencia ante la IVE, puede ser el tipo de país donde se presente esta solicitud. Lo anterior, en el sentido de que muy probablemente en un país desarrollado el negarse a practicar una IVE no pondrá en riesgo la vida de la mujer que la solicita, mientras que en un país de los menos desarrollados muy probablemente sí $\left({ }^{17}\right)$. Esta podría ser una limitación a la objeción de conciencia ante la IVE en los países menos desarrollados, como sería el caso de la América Latina.

Por otro lado, apoyando esta idea, en EE UU, Blustein y Fleischman, del American College of Obstetricians \& Gynecologists, han propuesto que, un médico con fuertes convicciones de naturaleza pro-vida no debería de practicar la medicina perinatal moderna, debido a que dentro de las posibilidades u opciones que existen al evaluar un embarazo, su interrupción puede ser una alternativa $\left({ }^{18}\right)$.

El caso mexicano se vuelve complejo, porque cada estado (provincia) es autónomo y cuenta con un código penal propio. De esta forma, el ordenamiento jurídico contempla 32 códigos penales. Casamadrid dice, en el caso mexicano, que "tratándose de aborto autorizado a consecuencia de una violación, el facultativo podría excusarse de practicarlo en razón de objeción de conciencia", y que "cuando se trate de un aborto necesario, en tanto urgencia médica, no es válida la objeción de conciencia del médico" $\left({ }^{19}\right)$. Sin embargo, se podría hacer algunas precisiones. Una de ellas es que, si bien México es una nación latinoamericana, también es cierto que el mosaico que existe en función de la estructura de su desarrollo estatal y de cada ciudad es enorme. Derivado de lo anterior, existen ciudades muy grandes con muchas opciones de atención, tanto pública como privada, así como poblaciones muy limitadas, en donde en ocasiones existe solamente un médico. Para lo que Casamadrid llama 'aborto necesario' habría que pensar también que la urgencia médica puede ser absoluta y relativa, y no sería igual que se presentara en una gran ciudad que en una comunidad pequeña. Las mismas reflexiones pueden extenderse al caso de la violación, la cual puede ocurrir en un estado donde exista 
como causal de despenalización, pero dentro de una comunidad pequeña, con un médico único. Las consideraciones no pueden ser tan generales, como lo hace Casamadrid; deben de ser individualizadas caso por caso.

\section{REFERENCIAS BIBLIOGRÁFICAS}

1. León Correa FJ. Fundamentos ético-jurídicos de la objeción de conciencia de los profesionales de la salud. Rev CONAMED. 2007;12(1):3-8.

2. Lee JM, Sotelo Monroy G, Casamadrid O. La objeción de conciencia en la práctica del médico. Rev Fac Med UNAM. 2006;49(3):121-5.

3. Arrieta JI. Objeción de conciencia. Las objeciones de conciencia a la ley y las características de su estructura jurídica. Cuadernos del Instituto de Investigaciones Jurídicas UNAM. 1998;3:27-55.

4. Fernández-Guardiola A. De cómo la conciencia es un proceso que comienza con una conversión analógica-digital y termina, probablemente, con una digital analógica, integrada por los genes, la percepción y la memoria. Salud Ment. 1996;19(2):50-6.

5. Orozco Cabal LF. Sobre la naturaleza humana. Explicación y comprensión de la conciencia. Rev Col Psiquiat. 2000;29:375-84.

6. De la Fuente R. El estudio de la conciencia: Estado actual. Salud Ment 2002;25(5):1-9.

7. Patiño Román A. Una disertación sobre la conciencia. Neurol Neurocir Psiquiat. 2003;36(4):202-5.

8. Díaz JL. La ordenación piramidal del cerebro y el enjambre de la conciencia. Primera parte. Salud Ment. 2006;29(2):712.

9. Díaz JL. La ordenación piramidal del cerebro y el enjambre de la conciencia. Segunda parte. Salud Ment. 2006;29(3):110
10. Ruiz Rodríguez V. La objeción de conciencia. Jurídica. Anuario del Departamento de derecho de la Universidad Iberoamericana. 2002;32:509-17.

11. Pacheco Escobedo A. Ley y conciencia. Derechos Humanos. 2002;54:61-8.

12. Gracia D. Fundamentos de Bioética. Madrid: Eudema; 1989.

13. Comisión Nacional de Bioética. Código de Bioética del Personal de Salud. México: Comisión Nacional de Bioética; 2002.

14. Comisión Nacional de Bioética. Código de Conducta para el Personal de Salud. México: Comisión Nacional de Bioética; 2002.

15. Marshall J, Raynor M. Conscientious objection 1: Legal and ethical issues. Br J Midwifery. 2002;10(6):389-92.

16. Raynor M, Marshall J. Conscientious objection 2: Professional responsibilities. $\mathrm{Br} \mathrm{J}$ Midwifery. 2002;10(9):574-7.

17. Van Bogaert LJ. The limits of conscientious objection to abortion in developing world. Developing World Bioeth. 2002;2(2):131-43.

18. Blustein J, Fleischman AR. The pro-life maternal-fetal medicine physician. Hastings Cent Rep. 1995;25(1):22-6.

19. Casamadrid Mata O. Objeción de conciencia. Objeción de conciencia en el derecho sanitario mexicano. Instituto de Investigaciones Jurídicas. México, DF: UNAM; 1998. p. 215-27.

Manuscrito recibido el 12 de enero de 2007 y aceptado para publicación el 01 de marzo de 2007.

\section{Correspondencia:}

Jorge Alberto Álvarez Díaz

Universidad Complutense de Madrid

Ciudad Universitaria, Facultad de Medicina

Plaza de Ramón y Cajal s/n

Departamento de Historia de la Medicina, Pabellón IV, Sótano,

Despacho 3. CP 28040, Madrid, España.

correo-e:bioetica_reproductiva@hotmail.com 